\title{
Proposta de um Modelo de e-Learning Social
}

\author{
José Martins ${ }^{1,2}$, Ramiro Gonçalves ${ }^{1,2}$, Vítor Santos ${ }^{3}$, Manuel Pérez Cota ${ }^{4}$, Tiago Oliveira 3 , \\ Frederico Branco ${ }^{1,2}$ \\ jmartins@utad.pt, ramiro@utad.pt, vsantos@isegi.unl.pt, mpcota@uvigo.es, toliveira@isegi. \\ unl.pt, fbranco@utad.pt \\ ${ }^{1}$ Universidade de Trás-os-Montes e Alto Douro, Vila Real - Portugal \\ ${ }^{2}$ INESC TEC, Universidade do Porto, Porto - Portugal \\ ${ }^{3}$ ISEGI - Universidade Nova de Lisboa, Lisboa - Portugal \\ ${ }^{4}$ Universidade de Vigo, Vigo - Spain
}

DOI: 10.17013/risti.16.92-107

Resumo: Compreendendo a cada vez maior necessidade de implementação de processos de aprendizagem sociais e colaborativos, analisámos os modelos pedagógicos e de ensino existentes, objetivando assim identificar uma base comum que pudesse suportar um curso de e-learning implementado sobre uma rede social. Como complemento, foi também realizada uma revisão da literatura sobre a adoção e uso de redes sociais, focando-nos na sua adoção para fins educativos, com o intuito de atingir as variáveis mais relevantes para a implementação de atividades de ensino em s-learning. A combinação destas atividades resultou na apresentação de um novo modelo de avaliação da aprendizagem, projetado para ser aplicado sobre cursos de e-learning implementados sobre o Facebook. Com este propósito em mente, é apresentada neste artigo uma descrição do modelo de avaliação proposto, focando a caracterização detalhada do processo de avaliação, atividades e tecnologias inerentes. No âmbito do presente projeto, foi também possível perceber que a execução de atividades pedagógicas e de investigação executadas sobre redes sociais são uma solução para melhorar a disposição e empenho do aluno no processo de aprendizagem.

Palavras-chave: e-Learning; Redes Sociais; s-Learning; Avaliação em s-Learning; Facebook.

\section{A Proposal for a Social e-Learning Model}

Abstract: By understanding the need for a social, collaborative and participative learning process, an analysis to the existent learning and pedagogical models was made in order to identify the existence of a basis for the development of an e-learning course implemented over a social network site. As a complement, a systematic literature review on social media adoption and use was made, particularly focusing the adoption for educational purposes and aiming at achieving the set of variables which might impact the implementation of s-learning activities. The combination of these activities resulted in the presentation of a new learning assessment model 
designed to be applied over e-learning courses implemented on social media. With this in mind, a description on the referred assessment model is presented, thus considering the need for a comprehensive characterization of the inherent assessment process and inherent activities and technologies. Through the execution of the research and pedagogical activities performed in the scope of the present project it was also possible to acknowledge that learning activities performed over social networks sites are a solution for improving student's willingness and commitment towards studying and learning.

Keywords: e-Learning; Social Media; s-Learning; s-Learning Assessment; Facebook.

\section{Introdução}

O desenvolvimento tecnológico é cada vez mais uma realidade dos tempos modernos a que nós, como indivíduos e sociedades, temos de nos adaptar, especialmente, quando se aplicam novas tecnologias a programas de aprendizagem escolar, extrapolando a importância do ambiente e-learning, por meio do qual milhares de estudantes aprendem todos os dias por todo o mundo (Tondeur et al., 2012). A associação de tecnologias com as atividades de aprendizagem foi desencadeando uma evolução significativa em termos do que é ensinado, como e onde é ensinado e avaliado e, também onde é aprendido. Esta afirmação torna-se mais verdadeira quando se examinam as tecnologias baseadas na Internet e as suas enormes vantagens para a implementação das atividades de e-learning, especialmente se forem consideradas as características sociais, colaborativas e participativas relacionadas com a maioria das tecnologias da Web 2.0 (Martins, Gonçalves, Santos, \& Pereira, 2012).

Ao analisar o grau de adoção das tecnologias relacionadas com a Web 2.0 atual é possível perceber que as redes sociais (RS) têm-se destacado como tendo o maior número de utilizadores ativos. (Martins, Gonçalves, \& Cota, 2011). Ao considerarmos os números de adoção das RS com a aceitação da sua natureza social, informal e interativa, surgiu a necessidade de realizarmos um estudo mais intensivo sobre como combinar as RS com atividades de e-learning, e particularmente atividades de avaliação, permitindo assim a conceção de novos modelos de aprendizagem que incorporam tecnologias Web mais interativas, ativas, colaborativas, cooperativas e sociais (Martins, Gonçalves, Oliveira, Pereira, \& Cota, 2014).

\section{Aprendizagem Social - Combinar e-Learning com Redes Sociais}

De acordo com Martins, Gonçalves, Pereira, and Cota (2012), o ritmo da sociedade atual cria a necessidade de implementação de sistemas que suportem iniciativas de aprendizagem à distância baseadas em ambientes de aprendizagem virtuais online.

\subsection{Conceitos e fundamentos do e-Learning}

Segundo a Agência de Garantia de Qualidade do Reino Unido para o Ensino Superior, o ensino à distância contempla o provisionamento de recursos de aprendizagem (materiais didáticos, ferramentas e orientação profissional) para o lugar onde o aluno está localizado, em vez de este se deslocar para instalações físicas tradicionais 
(QAA, 2008). A massificação dos dispositivos móveis em conjunto com o acesso a serviços de comunicação de dados mais acessíveis, transformou a ideia inicial de ensino à distância num moderno e tecnológico conceito com o nome "e-Learning", que é atualmente suportado por tecnologias como as plataformas Web e dispositivos móveis (Laouris \& Eteokleous, 2005).

A existência de vários agentes que se debruçam sobre as questões relacionadas com o e-learning tem levado a um desenvolvimento significativo do conceito e à implementação efetiva das várias iniciativas reais de e-learning com benefícios consideráveis para todas as partes envolvidas. Em termos de vantagens associadas ao e-learning, é possível destacar a facilidade com que os professores executam as tarefas, a flexibilidade e viabilidade inerente às metodologias de ensino e o planeamento dos curso e aulas, bem como resultados mais eficientes, principalmente porque o ritmo da aprendizagem pode ser definido pelo próprio estudante (Costa, Reis, \& Loureiro, 2014).

\subsection{Redes sociais - Caracterização da conceptualização e adoção}

Uma das tecnologias mais usadas e relevantes associadas com a Web 2.0 são as redes sociais (Kane, Alavi, Labianca, \& Borgatti, 2012). Mesmo que essas tecnologias se assumam a si mesmas como altamente inovadoras, os seus conceitos inerentes (grupo social) remontam aos primórdios dos estudos de psicologia e sociologia. As redes sociais podem ser definidas como aplicações de software destinadas a trabalhar na Web com o objetivo de promover e apoiar o estabelecimento de relações virtuais entre os indivíduos, entre as organizações e entre indivíduos e organizações (W. Kim, Jeong, \& Lee, 2010).

Ao analisar RS de um ponto de vista inovador e tecnológico, é possível perceber que a principal característica destas tecnologias não é a sua capacidade para promover e criar relações entre os utilizadores, mas a possibilidade destes mesmos utilizadores gerirem e tornarem visíveis as suas próprias redes de relações (Pallis, ZeinalipourYazti, \& Dikaiakos, 2011). Dito isto, pode-se supor que as RS impulsionam a criação de relações entre indivíduos que, num ambiente não-digital, não estabeleceriam qualquer tipo de ligação (Boyd \& Ellison, 2007). Desde o início do Séc. XXI, as RS têm florescido nos mercados mundiais de tecnologia, sendo a sua adoção massiva uma realidade amplamente aceite (Mergel, 2013).

As RS representam um tema muito atual, especialmente no que diz respeito à sua adoção, a nível pessoal e organizacional, como Martins et al. (2014) argumentam num artigo de revisão de literatura. Com isto em mente, e assumindo a estrutura/análise apresentada por Kane et al. (2012), na seção seguinte irá ser apresentada uma análise breve e muito focada sobre as variáveis cujo impacto para o processo de aprendizagem é mais significativo e que devem ser consideradas na criação de atividades de aprendizagem apoiados pelas RS.

\subsection{Como é que as redes sociais mudaram as atividades de aprendizagem}

Como referido por Siemens (2005), a existência de conhecimento é baseada na construção de redes de pares centrada em quem está a aprender. Com isto em mente, e apoiando as suas suposições no conceito de Ambiente de Aprendizagem Personalizada (AAP), Santos and Tavares (2014) alegam que o papel do professor é fundamental a fim 
de alcançar aprendizagens de qualidade, inclusive nos ambientes individuais visto que aqui podem ser desenhados novos cenários de aprendizagem onde os alunos utilizem ferramentas de comunicação e interação do seu quotidiano.

Enquanto nos ambientes de e-learning os professores devem agir simultaneamente como facilitadores, mentores e instrutores, o papel dos estudantes é mais simples tendo como objetivo a sua transformação em criadores e disseminadores ativos de conteúdos, e desta forma utilizam as iniciativas de e-learning não só para aprenderem mas também para desenvolverem a sua autonomia pessoal (Garrison, 2011).

Ao analisar as transformações sofridas pela Web ao longo da sua evolução para a versão 2.0, pode-se observar que nesta nova versão, os utilizadores têm uma participação ativa na criação colaborativa e atualização de conteúdo online, assumindo assim práticas que vão desde partilha ativa de documentos, vídeos e imagens, até à criação de conteúdo online através do uso de sistemas de colaboração (Downes, 2008). O desenvolvimento de projetos educacionais apoiados principalmente no uso de tecnologias Web 2.0, particularmente aquelas que incorporem componentes sociais, interativas e casuais, são propensas a gerar tendências motivacionais no sentido da sua utilização e sucesso inerente. A introdução de RS em currículos educacionais está a ser discutida por diversos autores como um assunto de fundamental importância e relevância para a modernização e adaptação das perspetivas educacionais tradicionais (Ebner, Lienhardt, Rohs, \& Meyer, 2010).

Apesar do potencial da Web 2.0 e das evidências associadas à sua adoção e uso, ainda há barreiras que se fazem sentir aquando do processo de adoção em contextos de aprendizagem (Greenhow, Robelia, \& Hughes, 2009): a falta de tempo para aprender a usar as tecnologias, a exigência de um retorno imediato, a falta de conhecimento sobre como usar e a inexistência de histórico nas vantagens inerentes ao uso. De acordo com Greenhow et al. (2009), existem três pensamentos principais por trás do sucesso das tecnologias Web 2.o utilizadas para fins educacionais: 1) o desenvolvimento das competências profissionais dos professores; 2) o desenvolvimento de metodologias para a criação de atividades de aprendizagem e conteúdos de qualidade; e 3) a necessidade de superar as assimetrias de aprendizagem.

Existem várias iniciativas que promovem a criação de comunidades de professores que incentivam às boas práticas e métodos para a utilização de tecnologias da Web 2.0 na sala de aula. No entanto, o impacto dessas iniciativas não tem sido significativo e sem falhas, indicando assim os enormes desafios que devem ser focados. Wilson and Ferreira (2010) indicam que a mudança necessária pode ser alcançada através do seguinte conjunto de novas estratégias: 1) a definição da escala adequada para reger a forma como as tecnologias da Web 2.0 são incorporadas nos cursos existentes; 2) a análise e reflexão sobre os fatores que motivam os alunos a usar tecnologias para fins educacionais; assegurar que os professores ganham consciência sobre as vantagens da utilização das tecnologias e que os mesmos tenham os conhecimentos técnicos e funcionais necessários para moderar e controlar as atividades de aprendizagem que utilizam ferramentas Web 2.0; 3) o correto alinhamento de estratégias educativas; e 4) permitir aos professores aderir a mecanismos através dos quais podem aprender boas práticas e partilhar as suas experiências, pensamentos e dúvidas. 
Vários investigadores têm estudado não só a adoção de RS a partir de um ponto de vista genérico, mas também a partir de uma perspetiva educacional (Martins, Goncalves, Branco, \& Peixoto, 2015). Ao analisarmos os números mais recentes, é possível observar que centenas de milhões de utilizadores da Internet já estão registados numa ou várias RS, representando esta situação um potencial enorme para a evolução em termos da evolução dos ambientes de ensino (Correa, Hinsley, \& De Zuniga, 2010; Dabbagh \& Kitsantas, 2012). Autores como Cheung, Chiu, and Lee (2011) reconhecem a existência de poucos estudos focados na adoção das RS, mas apesar desta constatação, os mesmos autores indicam que os estudos existentes apresentam uma análise muito complexa sobre os fatores influenciadores da adoção inicial, por parte dos alunos, das referidas tecnologias, alegando que a presença social, normas de grupos, relações sociais, propósitos de utilização para entretenimento e fatores sociais são as variáveis com mais influência no processo de adoção.

Com o objetivo de procurar indicadores sobre a importância do ambiente social, cultural e geográfico dos estudantes na adoção e uso de redes socias no ensino, Y. Kim, Sohn, and Choi (2011) elaboraram um estudo onde estudantes dos EUA e da Coreia do Norte foram analisados na tentativa de identificar os motivos pelos quais adotaram as RS e quais padrões de utilização destas tecnologias. Deste estudo foi possível perceber que a procura de amigos, apoio social, entretenimento, disponibilidade de informação e comodidade são os principais motivos para a adoção das RS em ambos os grupos de alunos.

Ajjan and Hartshorne (2008) realizaram um inquérito a mais de 130 membros do corpo docente de uma universidade dos Estados Unidos com o objetivo de perceber se as pessoas envolvidas nas atividades educativas consideram as RS úteis nessas mesmas atividades e, se sim, de que formas estas poderiam ser usadas. Com o trabalho destes autores foi possível reconhecer que mais de 50\% dos inquiridos consideram as RS como motivadores de interação aluno-aluno e que a utilização destas tecnologias se tornou muito dependente de fatores como a facilidade de uso, compatibilidade e perceção de utilidade.

Ao analisarmos os processos e atividades do e-learning, é relativamente simples perceber que a distribuição e partilha de conteúdos de aprendizagem é igualmente (se não mais) importante como o próprio conteúdo. De acordo com Chatti, Jarke, and Frosch-Wilke (2007), as tecnologias da Web 2.0, e especialmente plataformas como RS, podem permitir uma melhoria significativa da partilha de conhecimentos, bem como uma melhoria na performance da aprendizagem individual. Segundo Lin, Huang, and Chuang (2014), a criação de ambientes e-learning caracterizados por uma forte consciência de rede social e onde os alunos se sintam mais confortáveis na interação, terá impactos muito positivos sobre sucesso da aprendizagem do indivíduo e do grupo. Como se pôde constatar, o uso das RS como ferramentas para criar os ambientes indicados, força a existência de um paralelismo entre a informalidade do ambiente onde as pessoas se podem relacionar facilmente, partilhar conteúdo e pensamentos entre si, e entre a necessidade de existir um mecanismo de autorregulação através do qual as aprendizagens possam ser avaliadas em termos de aquisição individual de conhecimento e em termos de participação nas dinâmicas e interações do grupo.

Tendo em conta todas as reflexões efetuadas, reconhecemos a crescente necessidade para responder ao seguinte conjunto de hipóteses: 1) Quais são as variáveis associadas 
com a adoção das RS a nível individual que podem afetar o uso dessas tecnologias para apoiar atividades de aprendizagem? 2) De que forma as RS podem ser usadas como base tecnológica para atividades de e-learning? 3) Como se pode implementar processos de avaliação para atividades de aprendizagem suportadas pelas RS?

Na secção seguinte, apresentamos o modelo de e-learning social proposto e que serve como a base para responder aos desafios acima identificados.

\section{Modelo de e-Learning Social}

Como argumenta Dalsgaard (2006), para que o e-learning evolua, é preciso transcendêlo no sentido de uma realidade com um maior grau de independência em relação aos sistemas de gestão de aprendizagem.

\subsection{Redes sociais e e-Learning}

A especificidade das RS e os impactos que todos os dias estas tecnologias têm nos estudantes e professores durante a sua vida está a tornar-se cada vez mais significante. De acordo com vários autores, as RS estão a ser adotadas para fins educativos em ritmo semelhante ao verificado para outros fins (prazer, negócios, criação e gestão de relacionamentos, etc.), tornando esta tecnologia numa das que tem um futuro mais promissor nas iniciativas de e-learning (Mazman \& Usluel, 2010). Ao contrário do que se poderia pensar, a adoção das RS no apoio a turmas ou cursos de e-learning assume-se como um impulsionador para o desempenho dos professores e monitores, transmitindo assim aos alunos a noção de que o conhecimento não é aprendido simplesmente pela observação de um especialista, mas sim pela combinação desta atividade inicial com o estudo individual, discussão e interação do grupo (Baird \& Fisher, 2005; Mikroyannidis et al., 2013).

Como é argumentado por Wheeler and Wheeler (2009), as competências baseadas na educação e nos programas de treino efetivo são fatores determinantes para o futuro dos alunos e respetiva autorrealização. Na opinião de Dias, Diniz, and Hadjileontiadis (2014), o uso das RS é uma forma clara de cumprir com os fatores referidos, principalmente porque estas tecnologias permitem um processo de aprendizagem mais casual e interativo que pode ajudar, tanto os estudantes como os professores. De acordo com Shi, Al Qudah, and Cristea (2013) ao misturar os princípios do e-learning com as características e funcionalidades das RS, é possível criar programas educativos e aulas mais dinâmicas, que permitam aos professores definir metas de aprendizagem mais ativas onde os alunos sejam capazes de alcançar os objetivos definidos para as unidades curriculares através da utilização das RS para interação, partilha de conhecimentos pessoais, capacidades e materiais educativos.

Shi, Stepanyan, Al Qudah, and Cristea (2013) estudaram também como as interações dos alunos nas RS podem ser usadas como um fator de avaliação de aprendizagem, tendo atingido um conjunto de três tipos de âmbitos que podem ser utilizados para a avaliação das aprendizagens: 1) tipos de interações (Ex: posts, comentários, atualizações de status, mensagens, etc.), 2) número de interações e 3) a qualidade e complexidade das interações. 


\subsection{Descrição e caracterização do modelo social de e-learning proposto}

O modelo de e-learning social proposto baseia-se no pressuposto de que utilizar redes sociais servirá como um impulso para a instituição de relações informais de confiança e incentivar a interação entre alunos e entre alunos e professores. O modelo projetado garante não só a presença social, mas principalmente incentiva a que as normas dos grupos sejam uma realidade seguida por todos, permitindo assim a incorporação de vários tipos de atividades: 1) Atividades colaborativas - os alunos são divididos em grupos onde lhes é dado uma tarefa ou objetivo de aprendizagem que eles terão de discutir e resolver em grupo e para o qual serão também avaliados como um grupo (Fikret, 2012); 2) Atividades de cooperação - os alunos recebem uma tarefa ou objetivo de aprendizagem que terão de analisar, discutir e resolver através da interação com o grupo, mas para a qual serão avaliados individualmente (Slavin, 2011);3) atividades baseadas em problemas - é apresentado aos alunos um problema que eles têm que resolver. Estas atividades são feitas mediante o fornecimento de um conjunto de materiais de suporte e aprendizagem. A avaliação é feita individualmente (Dolmans, De Grave, Wolfhagen, \& Van Der Vleuten, 2005); 4) atividades ativas de aprendizagem - os alunos são o principal foco da atividade e são incentivados a analisar os materiais de aprendizagem, artigos técnicos e científicos, relatórios, conhecer bases de dados e interagir ativamente com os outros a fim de alcançar o know-how necessário para cumprir as metas pré-definidas da atividade; e 5) Troca ativa de funções - os alunos são divididos em grupos (e avaliadas como um grupo) onde é atribuído um tema que eles têm de analisar, desenvolver os materiais de aprendizagem necessários para um outro aluno entender o assunto e criar um questionário de avaliação também sobre esse mesmo tema. Depois de desenvolver estes objetivos, o grupo terá de apresentar aos restantes elementos da turma, interagindo entre si e com o "grupo de ensino", a fim de resolver todas as dúvidas e interpretações erradas.

Como pode ser observado na Figura 1, o fluxo do modelo de e-learning social proposto é composto por três fases: 1) uma fase inicial onde o professor não só é responsável pela criação do grupo da turma na RS (e convidar os alunos inerentes), mas também tem a função de apresentar à turma as principais metas e objetivos e proporcionar um teste

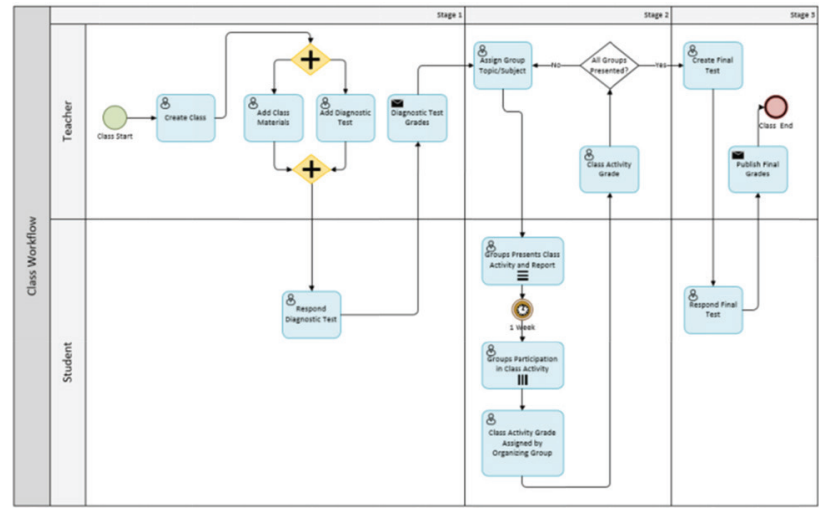

Figura 1 - Fluxo do modelo de e-learning social proposto representado de acordo com a notação BPMN. 
diagnóstico que os alunos têm de responder individualmente para que o professor fique ciente da profundidade do conhecimento do aluno sobre os temas da turma; 2) uma fase intermediária onde as atividades de e-learning social, prosseguem através de interações contínuas (e individuais) do grupo; e 3) a fase final, onde o professor apresenta a avaliação final que abrange todo o espectro de assuntos abordados que os alunos devem discutir isso entre si para apresentar uma resposta individual. Esta fase termina com a publicação das notas finais da turma e com o encerramento do curso.

Dada a natureza do objetivo proposto a equipa de investigação decidiu, de acordo com Fonseca et al. (2012), executar uma validação inicial, a fim de ser assegurada a validade do que está a ser apresentado. Com isto em mente, a implementação real do modelo proposto foi criada, os alunos e professores de uma universidade Portuguesa foram incorporados e vários indicadores qualitativos referidos foram recolhidos ao longo da implementação, a fim de assumir a validade mencionada anteriormente. Na Figura 2 pode-se visualizar os passos iniciais realizados quando foi criado o grupo na rede social "Facebook" (escolhida como ambiente virtual de aprendizagem), ao qual os alunos inscritos na unidade curricular de Plataformas Digitais (do Mestrado de Engenharia Informática da Universidade de Trás-os-Montes e Alto Douro) teriam de se associar. Nesta fase inicial, o professor responsável pela turma, cria o grupo no Facebook, descreve e caracteriza a forma global em que a turma irá trabalhar e apresenta as metas de aprendizagem expectáveis.

Para que as atividades letivas possam iniciar, o professor apresenta aos alunos um link para uma pasta especialmente criada num repositório online (Google Drive), que contém todos os materiais e recursos didáticos necessários para a fase inicial. Posteriormente, o professor terá de dividir os alunos em grupos e apresentar a atividade de avaliação diagnóstica que vão ter que concluir num curto período de tempo. Quando todos os alunos apresentam as suas respostas à referida atividade, o professor avalia a resposta de cada aluno e comunica individualmente a sua classificação identificado problemas e preocupações.

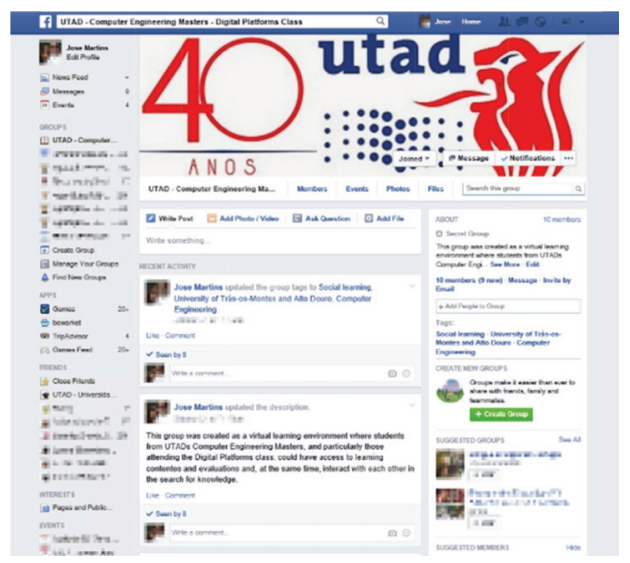

Figura 2 - Criação do grupo no Facebook que serviu como ambiente virtual de aprendizagem 
Quando a fase inicial termina, o professor atribui uma tarefa/tema a cada grupo de modo a que os seus elementos comecem a analisar e estudar o assunto, ganhando assim o conhecimento necessário para criar um conjunto de materiais de aprendizagem acerca do assunto, preparar um questionário de avaliação que os outros grupos irão responder e ser avaliados. Além de serem avaliados pelas respostas apresentadas pelos questionários de outros grupos, todos os estudantes serão também avaliados de acordo com a sua interação na página do Facebook da turma.

Depois de ser atribuída a todos os grupos de estudantes uma tarefa e, consequentemente, todos trabalharem nela e de apresentarem os resultados obtidos aos restantes grupos, o professor irá apresentar algumas considerações gerais de todo o espectro de temas que envolvem a turma e por fim apresentar aos alunos uma prova onde os temas e assuntos inerentes à turma estão descritos de uma forma mais completa e focada, os alunos devem responder a essa prova, a fim de ser elegíveis a receber aprovação.

Ao analisar os trabalhos de Radosevic, Orehovački, and Lovrenčic (2009) foi possível perceber que fatores como a atitude dos alunos para com a aprendizagem, o grau de facilidade, a compreensão do programa do curso/turma por parte dos alunos, a conceção dos programas e o planeamento das atividades, podem ter um impacto muito significativo sobre o desempenho dos alunos. Tendo em conta este problema, a equipa de investigação inerente ao presente trabalho, decidiu incorporar no modelo de ensino proposto, as seguintes preocupações de fundo: a) fornecer sempre materiais de aprendizagem de qualidade para que os alunos sejam capazes de obter o suporte teórico necessário; b) fornecer bons exemplos, completos e de qualidade para cada conceito ou assunto que está a ser transmitido; e c) recolher sempre o feedback dos alunos sobre o programa do curso/turma, estrutura e fluxo de trabalho, a fim de identificar possíveis falhas que devam ser melhoradas nas seguintes edições, visando assim a manutenção de bons níveis de qualidade.

\section{Processo de Avaliação do Modelo de e-Learning de Social}

Ao analisarmos de uma perspetiva global o modelo dee-learning social proposto, épossível identificar três grandes momentos de avaliação: 1) uma avaliação diagnóstica inicial, 2) uma fase de avaliação interativa resultante de um ciclo de atividades realizadas pelos diferentes grupos de estudantes, e 3) um momento de avaliação final mais abrangente onde o professor é capaz de avaliar o conhecimento que os alunos adquiriram.

\subsection{Fase 1 - Procedimento da avaliação diagnóstica inicial}

Quando o curso/turma inicia, o professor como seria de esperar, é alheio aos níveis de conhecimentos previamente adquiridos pelos seus alunos e quais são as suas perceções acerca dos temas e assuntos que vão ser analisados durante o curso. Com estas premissas aceites e verificadas, houve uma necessidade de incorporar no modelo de e-learning proposto, uma avaliação diagnóstica inicial que pode servir como uma fonte de informação em que professores podem confiar, não só para confirmar o seu planeamento inicial do curso/turma, mas também para fazer os ajustes a abordagens pedagógicas necessários na sua metodologia (Adodo, 2013). Ao considerarmos os argumentos apresentados anteriormente, a equipa de investigação decidiu planear e 
implementar uma atividade de avaliação diagnóstica composta por um instrumento de avaliação de escolha múltipla de dois níveis, focada nos temas e assuntos inerentes à turma, em que cada aluno, não só escolhe a resposta correta, mas também tem de justificá-la. Dada a natureza eletrônica do ambiente de aprendizagem, o instrumento de avaliação diagnóstica referido é criado pelo professor através de plataformas de inquéritos online (ex: Survey Monkey) ou plataformas de acesso livre online (ex: Google Docs, Microsoft Office Online) onde os alunos devem responder ao teste usando essa mesma plataforma.

Ao analisar os resultados da avaliação diagnóstica, os professores devem ter como objectivo não só reconhecer os níveis de especialização dos alunos sobre os temas e assuntos, mas também tentar identificar possíveis situações que podem requerer a necessidade de mais atenção (ex: alunos sem conhecimento nem consciência sobre os temas e/ou assuntos, ou estudantes com algum tipo de deficiência ou incapicadade de aprendizagem).

\subsection{Fase 2 - Procedimento da avaliação interativa}

Quando o curso/turma atinge a segunda fase do modelo de e-learning social proposto, será atribuída uma tarefa/assunto a todos os grupos de estudantes que eles têm de analisar, reconhecer e discutir a fim de serem capazes de desenvolver um conjunto de materiais de aprendizagem relacionados com o tema (ex: manuais, tutoriais multimédia, exemplos e exercícios práticos, etc.) que será usado pelos colegas, juntamente com um questionário de avaliação (alinhado com o material desenvolvido) com o objetivo de ser respondido individualmente pelos mesmos.

As atividades do grupo referidas são executadas de forma cíclica a cada semana onde um dado grupo assume o "papel do professor" e partilha os resultados do seu trabalho com a apresentação aos restantes grupos do questionário de avaliação criado usando plataformas de inquérito online ou com as plataformas de acesso livre online.

Durante esta fase intermédia, o professor do curso/turma vai assumir uma mentalidade de motivador e moderador, com o objetivo de desencadear a interação do aluno dentro do grupo da turma no Facebook, para que as tarefas e atividades de aprendizagem colaborativa, cooperativa e ativa sejam realizadas. Apesar desta posição mais linear no que diz respeito à turma, o professor tem de estar totalmente comprometido com um acompanhamento constante das interações dos alunos, com o objetivo de analisá-las e avaliá-las em quantidade e qualidade (Teixeira \& Azevedo, 2011). A fim de fazer isso, o professor teve de usar uma ferramenta de software (atualmente na versão de protótipo funcional) que analisa cada interação do aluno e retorna uma classificação individual que pode assumir um valor de o a 20. O software referido, desenvolvido de propósito durante o projeto da investigação inerente, integrado com uma API (Application Programming Interface) do próprio Facebook tem o objetivo de obter os dados analíticos necessários.

Para cada "ciclo de grupo", em que um dado grupo assume o papel de professor, o professor da turma não terá apenas de avaliar o que os outros grupos fazem e as suas respostas ao questionário de avaliação proposto, mas também os materiais, conteúdos e o consequente questionário criado pelo grupo no comando. Em suma, cada grupo é avaliado pelas suas interações e respostas a avaliações propostas por outros grupos, mas também pelas suas próprias "atividades de ensino”. 


\subsection{Fase 3 - Procedimento da avaliação final}

Depois de todos os grupos terem tido a oportunidade de analisar, discutir e apresentar determinado assunto para a restante turma, o professor irá criar algumas interações no grupo do Facebook para relembrar todos os temas/assuntos abordados durante o período de aula e, consequentemente, apresentar a todos os alunos o questionário individual de avaliação final que resume todos os temas/assuntos que foram focados no âmbito curso/ turma. A fim de seguir a mesma metodologia no que diz respeito às tecnologias utilizadas para avaliar a aprendizagem dos estudantes, o questionário de avaliação final é também construído e disponibilizado através da utilização de plataformas de inquéritos online ou plataformas de acesso livre online, permitindo, assim, atividades de avaliação mais estáveis e familiares.

De acordo com o modelo de e-learning social proposto, a classificação final do curso/ turma dos alunos (SFC) é composta por vários elementos, como é possível verificar na seguinte fórmula:

$$
S F C=\left(\frac{\left(\frac{\sum_{i=1}^{K} \theta(i)}{K}+\gamma\right)}{2} \times \frac{5}{10}\right)+\left(\delta \times \frac{3}{10}\right)+\left(\beta \times \frac{2}{10}\right)
$$

- “ “ $\theta$ " representa a classificação da resposta dos alunos para todos os questionários respondidos. Este elemento de avaliação tem uma natureza quantitativa e pode ser atribuído um valor de o a 20;

- “ “” representa o número total de (intermediário) questionários que cada aluno teve que responder;

- " $\gamma$ " representa a classificação do grupo de estudantes sobre a qualidade global e precisão científica/técnica dos materiais de aprendizagem desenvolvidos e questionários de avaliação inerentes. Este elemento de avaliação também tem uma natureza quantitativa e pode ser atribuído um valor de o a 20;

- “ $\delta$ " representa a nota obtida pelos alunos no final do questionário de avaliação apresentado pelo professor. Considerando o propósito deste elemento de avaliação, definiu-se como um elemento quantitativo que pode ser atribuído um valor de o a 20;

- “ $\beta$ ” representa o nível das interações realizadas pelo aluno no grupo do curso/ turma no Facebook. Este elemento de avaliação quantitativa também representa uma variável que pode ser atribuído um valor de o a 20.

Ao combinar todos os elementos de avaliação apresentados e as suas respetivas influências, o professor alcança o valor SFC, que é apresentado individualmente, e em detalhe, a cada aluno através de uma mensagem privada no Facebook, onde é apresentado globalmente (valor final) a todo o curso/turma através de um post no grupo do Facebook.

Tendo por base as constatações de Karim, S., and Hussin (2006), Holsapple and LeePost (2006) e Biggs (2011), e de forma a assegurar uma evolução continua do modelo 
de ensino proposto, foi definida uma abordagem de recolha ativa de feedback através de questionários (disponibilizados online), onde os alunos podem indicar a sua opinião acerca dos materiais e recursos disponibilizados, sobre as metodologias de ensino utilizadas, sobre o professor e sobre o funcionamento global do curso. No caso de estudo utilizado para validar inicialmente o modelo proposto, foi realizado um questionário anunciado aos alunos através de uma notificação no grupo do Facebook inerente à unidade curricular, e que eles puderam responder online e de forma anónima.

\section{Conclusões}

Ao analisar o espectro completo da tecnologia utilizada em atividades de aprendizagem é possível identificar bons exemplos: tais como o uso de tecnologias como ferramentas de criação, gestão e suporte de atividades e ambientes virtuais de aprendizagem. Através de uma revisão sistemática da literatura científica existente focada nos conceitos de e-learning e redes sociais e da forma em que eles se podem relacionar, foi possível reconhecer que, apesar de um conjunto de variáveis que afetam a adoção e utilização das RS, existem alguns estudos e pesquisas que visam o desenvolvimento de atividades de e-learning baseadas nas redes sociais.

Baseado nestas premissas apresentamos neste artigo um modelo de e-learning com recurso a redes sociais cujo objetivo central é combinar a familiaridade dos alunos com as redes sociais e um programa curricular definido para ser executado estritamente online. De acordo com o modelo proposto isto é realizado através de ações dinâmicas, colaborativas e altamente relacionais e interativas, minimizando assim o impacto da falta do fator humano, tantas vezes associado ao e-learning. O modelo proposto é composto por três fases: uma fase inicial onde os professores podem realizar uma avaliação diagnóstica inicial da consciência dos alunos sobre os temas do curso, uma fase intermediária onde os estudantes estão envolvidos numa série de atividades de aprendizagem ativa, colaborativa e cooperativa onde eles não só assumem o papel do aluno, mas também o papel do professor ao criar conteúdos de aprendizagem sobre temas e tópicos relacionados com o curso e uma fase final onde o professor realiza a avaliação de aprendizagem final.

Apesar da natureza dinâmica do modelo apresentado, foi também planeado e definido um processo de avaliação estruturado em três etapas. Esta avaliação incorpora uma avaliação inicial de diagnóstico (apoiado por um instrumento de avaliação de escolha múltipla de duas camadas), um conjunto de avaliações intermediárias com base num conjunto limitado de tópicos/assuntos anteriores, e por fim um questionário de avaliação que incorpora todos os tópicos/assuntos abordados no curso/turma. Todas as atividades de avaliação referidas devem ser implementadas através da utilização de tecnologias de questionários online que podem facilmente ser incorporados na página do grupo do curso/turma. A fim de validar o modelo proposto, foi feito um teste real numa universidade Portuguesa em que os alunos estavam envolvidos num curso totalmente implementado no Facebook, interagindo e colaborando entre si com uma atitude fervorosa e entregando um feedback muito positivo, não só na forma pela qual o curso foi realizado, mas também sobre a estratégia utilizada para a avaliação da sua aprendizagem. 
Em suma, com o presente trabalho, pretende-se contribuir ainda mais para o desenvolvimento e melhoria não só do e-learning social, mas também das suas atividades inerentes, processos de avaliação e tecnologias. Ainda assim, e assumindo a existência de variáveis e condicionantes que devem ser controlados de forma mais assumida, futuramente será objetivo que o presente projeto seja novamente replicado, agora em mais do que uma unidade curricular, permitindo assim que mais professores possam colaborar e assumindo também a participação de especialistas do foro educacional e psicológico, como elementos diferenciadores e incorporadores de perspetivas diferentes, mas ao mesmo tempo, complementares.

\section{Referências}

Adodo, S. (2013). Effects of Two-Tier Multiple Choice Diagnostic Assessment Items on Students' Learning Outcome in Basic Science Technology (BST). Academic Journal of Interdisciplinary Studies, 2(2), 201-210.

Ajjan, H., \& Hartshorne, R. (2008). Investigating faculty decisions to adopt Web 2.0 technologies: Theory and empirical tests. The Internet and Higher Education, 11(2), 71-80. doi: doi:10.1016/j.iheduc.2008.05.002

Baird, D., \& Fisher, M. (2005). Neomillennial user experience design strategies: Utilizing social networking media to support "always on" learning styles. Journal of educational technology systems, 34(1), 5-32.

Biggs, J. (2011). Teaching for quality learning at university: What the student does: McGraw-Hill Education (UK).

Boyd, D., \& Ellison, N. (2007). Social Network Sites: Definition, History, and Scholarship. Journal of Computer-Mediated Communication, 13(1), \#11.

Chatti, M., Jarke, M., \& Frosch-Wilke, D. (2007). The future of e-learning: a shift to knowledge networking and social software. International Journal of Knowledge and Learning, 3(4), 404-420.

Cheung, C., Chiu, P., \& Lee, M. (2011). Online social networks: Why do students use facebook? Computers in Human Behavior, 27(4), 1337-1343. doi: doi:10.1016/j. chb.2010.07.028

Correa, T., Hinsley, A., \& De Zuniga, H. (2010). Who interacts on the Web?: The intersection of users' personality and social media use. Computers in Human Behavior, 26(2), 247-253.

Costa, A., Reis, L., \& Loureiro, M. (2014). Desenvolvimento de Software Educativo: A Coordenação como Fator Crítico de Sucesso. RISTI-Revista Ibérica de Sistemas e Tecnologias de Informação(SPE2), 1-18.

Dabbagh, N., \& Kitsantas, A. (2012). Personal Learning Environments, social media, and self-regulated learning: A natural formula for connecting formal and informal learning. The Internet and Higher Education, 15(1), 3-8. doi:10.1016/j. iheduc.2011.06.002 
Dalsgaard, C. (2006). Social software: E-learning beyond learning management systems. European Journal of Open, Distance and E-Learning, 2006(2).

Dias, S., Diniz, J., \& Hadjileontiadis, L. (2014). E-Learning Exequibility in the Information and Knowledge Society Towards an Intelligent Learning Management System Under Blended Learning (Vol. 59, pp. 3-19): Springer International Publishing.

Dolmans, D., De Grave, W., Wolfhagen, I., \& Van Der Vleuten, C. (2005). Problembased learning: Future challenges for educational practice and research. Medical education, 39(7), 732-741.

Downes, S. (2008). Places to go: Connectivism \& connective knowledge. Innovate: Journal of Online Education, 5(1).

Ebner, M., Lienhardt, C., Rohs, M., \& Meyer, I. (2010). Microblogs in Higher Education-A chance to facilitate informal and process-oriented learning? Computers \& Education, 55(1), 92-100.

Fikret, T. (2012). Current situation and analysis of geography teachers active learning knowledge and usage in Turkey. Educational Research and Reviews, 7(18), 393-400.

Fonseca, B., Pereira, Â., Sanders, R., Barracho, V., Lapajne, U., Rus, M., . . Bojovic, V. (2012). PLAYER-a European Project and a Game to Foster Entrepreneurship Education for Young People. J. UCS, 18(1), 86-105.

Garrison, D. R. (2011). E-learning in the 21st century: A framework for research and practice: Taylor \& Francis.

Greenhow, C., Robelia, B., \& Hughes, J. E. (2009). Learning, teaching, and scholarship in a digital age Web 2.0 and classroom research: What path should we take now? Educational Researcher, 38(4), 246-259.

Holsapple, C., \& Lee-Post, A. (2006). Defining, Assessing, and Promoting E-Learning Success: An Information Systems Perspective*. Decision Sciences Journal of Innovative Education, 4(1), 67-85. doi:10.1111/j.1540-4609.2006.00102.x

Kane, G., Alavi, M., Labianca, G., \& Borgatti, S. (2012). What's different about social media networks? A framework and research agenda. MIS quarterly.

Karim, N., S., D., \& Hussin, R. (2006). Mobile phone applications in academic library services: a students' feedback survey. Campus-Wide Information Systems, 23(1), 35-51. doi:doi:10.1108/10650740610639723

Kim, W., Jeong, O., \& Lee, S. (2010). On social Web sites. Information Systems, 35(2), 215-236. doi:10.1016/j.is.2009.08.003

Kim, Y., Sohn, D., \& Choi, S. (2011). Cultural difference in motivations for using social network sites: A comparative study of American and Korean college students. Computers in Human Behavior, 27(1), 365-372. doi: 10.1016/j.chb.2010.08.015

Laouris, Y., \& Eteokleous, N. (2005). We need an educationally relevant definition of mobile learning. Paper presented at the Proceedings of the 4th World Conference on Mobile Learning. 
Lin, J., Huang, H., \& Chuang, Y. (2014). The impacts of network centrality and selfregulation on an e-learning environment with the support of social network awareness. British Journal of Educational Technology, n/a-n/a. doi:10.1111/ bjet.12120

Martins, J., Goncalves, R., Branco, F., \& Peixoto, C. (2015, 17-20 June 2015). Social networks sites adoption for education: A global perspective on the phenomenon through a literature review. Paper presented at the 2015 10th Iberian Conference on Information Systems and Technologies (CISTI), Águeda, Portugal. doi:10.1109/ CISTI.2015.7170490

Martins, J., Gonçalves, R., \& Cota, M. (2011). Web 2.o Business Impact. Paper presented at the CISTI2011 - $6^{\text {a }}$ conferência Ibérica de Sistemas e Tecnologias de Informação, Chaves, Portugal.

Martins, J., Gonçalves, R., Oliveira, T., Pereira, J., \& Cota, M. (2014). Social networks sites adoption at firm level: A literature review. Paper presented at the CISTI2014Conferência Ibérica de Sistemas e Tecnologias de Informação, Barcelona, Espanha.

Martins, J., Gonçalves, R., Pereira, J., \& Cota, M. (2012, 20-23 June 2012). Iberia 2.o: A way to leverage Web 2.o in organizations. Paper presented at the 7 th Iberian Conference on Information Systems and Technologies (CISTI 2012), Madrid, Spain.

Martins, J., Gonçalves, R., Santos, V., \& Pereira, J. (2012). Network Based Model For E-Learning 2.0. Procedia - Social and Behavioral Sciences, $47(0), 1242-1248$. doi:10.1016/j.sbspro.2012.06.807

Mazman, S., \& Usluel, Y. (2010). Modeling educational usage of Facebook. Computers \& Education, 55(2), 444-453. doi:10.1016/j.compedu.2010.02.008

Mergel, I. (2013). Social media adoption and resulting tactics in the U.S. federal government. Government Information Quarterly, 30(2), 123-130. doi:10.1016/j. giq.2012.12.004

Mikroyannidis, A., Okada, A., Scott, P., Rusman, E., Specht, M., Stefanov, K., ... Hetzner, S. (2013). weSPOT: A Personal and Social Approach to Inquiry-Based Learning. J. UCS - Journal of Universal Computer Science, 19(14), 2093-2111.

Pallis, G., Zeinalipour-Yazti, D., \& Dikaiakos, M. (2011). Online Social Networks: Status and Trends. In A. Vakali \& L. C. Jain (Eds.), New Directions in Web Data Management (Vol. 331, pp. 213-234): Springer.

QAA. (2008). Outcomes from institutional audit: Institutions' support for elearning Quality Assurance Agency for Higher Education.

Radosevic, D., Orehovački, T., \& Lovrenčic, A. (2009). Verificator: educational tool for learning programming. Informatics in Education-An International Journal (Vol 8_2), 261-280.

Santos, V., \& Tavares, C. (2014). Implementation model for social learning. Paper presented at the 9th Iberian Conference on Information Systems and Technologies (CISTI 2014), Barcelona, Spain. 
Shi, L., Al Qudah, D., \& Cristea, A. (2013). Social e-learning in topolor: a case study.

Shi, L., Stepanyan, K., Al Qudah, D., \& Cristea, A. (2013). Evaluation of social interaction features in topolor-a social personalized adaptive e-learning system. Paper presented at the Proceedings of 13th IEEE International Conference on Advanced Learning Technologies (ICALT 2013).

Siemens, G. (2005). Connectivism: A learning theory for the digital age. International journal of instructional technology and distance learning, 2(1), 3-10.

Slavin, R. (2011). Cooperative learning. Learning and Cognition in Education Elsevier Academic Press, Boston, 160-166.

Teixeira, D., \& Azevedo, I. (2011). Análise de opiniões expressas nas redes sociais. RISTI-Revista Ibérica de Sistemas e Tecnologias de Informação(8), 53-65.

Tondeur, J., van Braak, J., Sang, G., Voogt, J., Fisser, P., \& Ottenbreit-Leftwich, A. (2012). Preparing pre-service teachers to integrate technology in education: A synthesis of qualitative evidence. Computers \& Education, 59(1), 134-144. doi:10.1016/j. compedu.2011.10.009

Wheeler, S., \& Wheeler, D. (2009). Using wikis to promote quality learning in teacher training. Learning, Media and Technology, 34(1), 1-10.

Wilson, T., \& Ferreira, G. (2010). Investigating the use of web 2.0 tools and Open Educational Resources for ICS. 